\title{
Approximating the inverse of banded matrices by banded matrices with applications to probability and statistics
}

\author{
Peter Bickel and Marko Lindner
}

February 24, 2010

\begin{abstract}
In the first part of this paper we give an elementary proof of the fact that if an infinite matrix $A$, which is invertible as a bounded operator on $\ell^{2}$, can be uniformly approximated by banded matrices then so can the inverse of $A$. We give explicit formulas for the banded approximations of $A^{-1}$ as well as bounds on their accuracy and speed of convergence in terms of their band-width. In the second part we apply these results to covariance matrices $\Sigma$ of Gaussian processes and study mixing and beta mixing of processes in terms of properties of $\Sigma$. Finally, we note some applications of our results to statistics.
\end{abstract}

Mathematics subject classification (2000): 60G15; 47B36, 47L10, 62H25, 62M10, 62M20.

Keywords and phrases: infinite band-dominated matrices, Gaussian stochastic processes, mixing conditions, high dimensional statistical inference.

\section{Introduction}

Let $\mathcal{I}$ be either the set of natural numbers, $\mathbb{N}$, or the integers, $\mathbb{Z}$, and let $\ell^{2}$ denote the corresponding Hilbert space of one- or two-sided infinite sequences $\left(u_{k}\right)_{k \in \mathcal{I}}$ of complex numbers with $\sum_{k \in \mathcal{I}}\left|u_{k}\right|^{2}<\infty$. We know that every bounded linear operator $A$ on $\ell^{2}$ can naturally be identified with a (one- or two-sided) infinite matrix $\left(a_{i j}\right)_{i, j \in \mathcal{I}}$. We will therefore use the words 'operator' and 'matrix' synonymously here.

It is clear that the inverse of a banded infinite matrix $A$, if it exists, is in general not a banded matrix any more. However, one can show that it is still the uniform limit of a sequence of such, that is: it is what we call a band-dominated matrix. By a simple approximation argument, this result immediately implies that the class of band-dominated matrices is inverse closed, that means: if one of them is invertible, its inverse is again band-dominated.

Outline of the Paper. We will give a proof of this result in Section 2 which moreover comes with explicit formulas for the banded approximations of the inverse and with bounds on their accuracy and speed of convergence in terms of their band-width. In Section 3 we apply these formulas to another class of operators, the so-called Wiener algebra. These inverse closedness results themselves are not new (see e.g. Kurbatov $[9,10,11]$ but also $[12,13,16,19,20,17,14]$ for related questions) but what we believe is new here is our approach and the explicit approximates of the inverse that it comes with, as well as the generalizations of our results to the operator classes defined in Section 4 with an eye to applications in statistics.

Next, in Section 5, we study the relation between our results in Sections 2 and 3 and the notion of regularity in Gaussian processes, a well settled problem in the stationary case, clarified, as we believe, for the first time in the general (non-stationary) case. In the same section, we consider the characterization of the notion of beta mixing (or absolute regularity) for Gaussian processes, a problem considered by Ibragimov and Solev [6] for the stationary case. We give a necessary and sufficient condition for beta mixing in the general case, relating it to closure notions stronger than those in the previous sections. Finally, in Section 6, we sketch the applications to statistics which initially prompted this work. 
Notations. Here and in what follows, let $\ell^{2}:=\ell^{2}(\mathcal{I})$ stand for the set of all sequences $x=\left(x_{k}\right)_{k \in \mathcal{I}}$ of complex numbers with

$$
\|x\|:=\sqrt{\sum_{k \in \mathcal{I}}\left|x_{k}\right|^{2}}<\infty
$$

where the index set $\mathcal{I}$ is fixed. For every bounded and linear operator $A$ on $\ell^{2}$, let $A^{*}$ denote the adjoint operator with matrix representation $\left(a_{i j}\right)^{*}=\left(\overline{a_{j i}}\right)$ and let $\|A\|$ denote the induced operator norm, that is

$$
\|A\|=\sup _{x \in \ell^{2} \backslash\{0\}} \frac{\|A x\|}{\|x\|} .
$$

By putting $\mathcal{I}=\{1, \ldots, n\}$ in the above, we define $\|x\|$ and $\|A\|$ as is usual for finite vectors $x \in \mathbb{C}^{n}$ and $n \times n$ matrices $A$ acting on them. We will now suppose that $\mathcal{I} \in\{\mathbb{Z}, \mathbb{N}\}$.

Let $\mathrm{BL}:=\mathrm{BL}\left(\ell^{2}\right)$ be the set of all bounded linear operators $A: \ell^{2} \rightarrow \ell^{2}$. Equipped with addition, multiplication by scalars, operator composition and the above norm, BL is a Banach algebra and even a $C^{*}$-algebra with involution $A \mapsto A^{*}$. If $A \in \mathrm{BL}$ is invertible (i.e. a bijection $\ell^{2} \rightarrow \ell^{2}$ ) then also $A^{-1} \in \mathrm{BL}$ as a consequence of the open mapping theorem. Now let $\mathrm{BO}:=\mathrm{BO}\left(\ell^{2}\right)$ refer to the set of all operators $A \in \mathrm{BL}$ that are induced by a banded matrix - meaning a matrix with only finitely many nonzero diagonals. Clearly, the set BO is closed under addition, multiplication, multiplication by scalars and under passing to the adjoint - but it is not closed in the operator norm $\|\cdot\|$ on BL. That is why one is interested in the norm closure of $\mathrm{BO}$, henceforth denoted by $\mathrm{BDO}:=\mathrm{BDO}\left(\ell^{2}\right)$, the elements of which are called band-dominated operators/matrices.

From a computational point of view the operator norm $\|\cdot\|$ is not very handy. An alternative norm $\llbracket \cdot \rrbracket$ can be defined on $\mathrm{BO}$ as follows: For $A \in \mathrm{BO}$ with matrix representation $\left(a_{i j}\right)_{i, j \in \mathcal{I}}$ and for each $k \in \mathbb{Z}$, let $d_{k}$ be the supremum norm of the $k$-th diagonal of $A$, that is

$$
d_{k}:=\sup \left\{\left|a_{i j}\right|: i, j \in \mathcal{I}, i-j=k\right\}, \quad \text { and put } \quad \llbracket A \rrbracket:=\sum_{k \in \mathbb{Z}} d_{k} .
$$

It is easy to see that this defines a norm on $\mathrm{BO}$ with $\|A\| \leq \llbracket A \rrbracket$ for all $A \in \mathrm{BO}$. Let us this time pass to the completion of $\mathrm{BO}$ in the stronger norm $\llbracket \cdot \rrbracket$; what we get is a proper subset of $\mathrm{BDO}$ that shall be denoted by $\mathcal{W}$. Equivalently, $A \in \mathcal{W}$ iff $\llbracket A \rrbracket<\infty$, where $\llbracket A \rrbracket$ is as defined in (1) but now for arbitrary infinite matrices. It turns out that $(\mathcal{W}, \llbracket \cdot \rrbracket)$ is a Banach algebra that is often referred to as the Wiener algebra. If we, for a moment, generalize our setting from operators on $\ell^{2}$ to operators on $\ell^{p}$ with $p \in[1, \infty]$, it is clear that the class $\mathrm{BO}$ does not depend on $p$. Unlike the norm closure $\mathrm{BDO}\left(\ell^{p}\right)$ of $\mathrm{BO}$, the Wiener algebra $\mathcal{W}$ is also independent of $p$ since it is defined merely in terms of matrix entries. One has, for all $p \in[1, \infty]$, that

$$
\mathcal{W} \subset \operatorname{BDO}\left(\ell^{p}\right) \quad \text { with } \quad\|A\| \leq \llbracket A \rrbracket
$$

for all $A \in \mathcal{W}$. We will give an elementary proof of the inverse closedness of $\mathcal{W}$, thereby automatically proving Wiener's famous theorem on functions with absolutely summable Fourier series.

\section{BDO is inverse closed}

The shortest proof of the inverse closedness of BDO goes like this: By its definition, BDO is a Banach subalgebra of $\mathrm{BL}$ that is closed under the involution map $A \mapsto A^{*}$. Since, moreover, the equality $\left\|A^{*} A\right\|=\|A\|^{2}$ holds for all $A \in \mathrm{BL}$, both $\mathrm{BL}$ and $\mathrm{BDO}$ are $\mathrm{C}^{*}$-algebras, and a basic theorem [4] on $\mathrm{C}^{*}$-algebras says that therefore $\mathrm{BDO}$ is inverse closed in $\mathrm{BL}$, i.e. if $A \in \mathrm{BDO}$ is invertible in $\mathrm{BL}$ one always has $A^{-1} \in \mathrm{BDO}$. In this section we will find out how to approximate $A^{-1}$ by band matrices and how good this approximation is.

In order to distinguish between banded matrices of different band widths $k \in \mathbb{N}_{0}:=\mathbb{N} \cup\{0\}$, we will introduce the notation $\mathrm{BO}_{k}$ for the set of all $A \in \mathrm{BL}$ whose matrix $\left(a_{i j}\right)_{i, j \in \mathcal{I}}$ is supported on the 
diagonals numbered $-k, \ldots, k$ only, that means $a_{i j}=0$ if $|i-j|>k$, i.e. $d_{n}=0$ if $|n|>k$ with $d_{n}$ from (1). Clearly, we have $\mathrm{BO}_{k} \subset \mathrm{BO}_{k+1}, \mathrm{BO}=\cup_{k \geq 0} \mathrm{BO}_{k}$ and

$$
A \in \mathrm{BDO} \quad \text { iff } \quad 0=\operatorname{dist}(A, \mathrm{BO})=\operatorname{dist}\left(A, \bigcup_{k=0}^{\infty} \mathrm{BO}_{k}\right)=\lim _{k \rightarrow \infty} \operatorname{dist}\left(A, \mathrm{BO}_{k}\right)
$$

with the usual definition of the distance, $\operatorname{dist}(A, S):=\inf _{B \in S}\|A-B\|$, of an operator $A \in$ BL from a set $S \subset \mathrm{BL}$. Note that if $a_{i j}$ is a matrix entry of $A$ with $|i-j|>k$ then clearly $a_{i j}$ is still a matrix entry of $A-B$ for all $B \in \mathrm{BO}_{k}$ so that $\|A-B\| \geq\left|a_{i j}\right|$. Consequently,

$$
\operatorname{dist}\left(A, \mathrm{BO}_{k}\right)=\inf _{B \in \mathrm{BO}_{k}}\|A-B\| \geq\left|a_{i j}\right|, \quad|i-j|>k
$$

holds, i.e. $\operatorname{dist}\left(A, \mathrm{BO}_{k}\right)$ is a bound on all matrix entries outside the $-k, \ldots, k$ band of $A$. Using the diagonal suprema introduced in (1), we can rephrase this as

$$
\operatorname{dist}\left(A, \mathrm{BO}_{k}\right) \geq \sup \left\{\left|a_{i j}\right|: i, j \in \mathcal{I}, i-j=n\right\}=d_{n}, \quad|n|>k .
$$

We start with the simple case when $A$ is banded and self-adjoint positive definite, i.e. $A \in \mathrm{BO}, A=A^{*}$ and the spectrum of $A, \operatorname{sp} A$, is strictly positive. In this case, it is well known that

$$
M:=\sup _{\lambda \in \operatorname{sp} A}|\lambda|=\varrho(A)=\|A\|, \quad m:=\inf _{\lambda \in \operatorname{sp} A}|\lambda|=1 / \varrho\left(A^{-1}\right)=1 /\left\|A^{-1}\right\|
$$

with $\varrho(A)$ denoting the spectral radius of $A$. Moreover, we have that $\kappa:=M / m=\|A\|\left\|A^{-1}\right\|$ is the condition number of $A$.

Lemma 2.1 Let $A \in \mathrm{BO}_{k}$ for some $k \in \mathbb{N}_{0}$ be self-adjoint positive definite, and define $M, m$ and $\kappa$ as above. Then, for every $n \in \mathbb{N}_{0}$, it holds that

$$
\operatorname{dist}\left(A^{-1}, \mathrm{BO}_{n \cdot k}\right) \leq \frac{1}{m}\left(\frac{M-m}{M+m}\right)^{n+1}=\frac{1}{m}\left(\frac{\kappa-1}{\kappa+1}\right)^{n+1}
$$

where an approximation $B_{n} \in \mathrm{BO}_{n \cdot k}$ of $A^{-1}$ with this accuracy is given in (6) below. In particular, $A^{-1} \in \mathrm{BDO}$ since the right-hand side of (5) goes to zero as $n \rightarrow \infty$.

Proof. We start by looking for a $\gamma \in \mathbb{R}$ such that

$$
\|I-\gamma A\|=\varrho(I-\gamma A)=\sup _{\lambda \in \operatorname{sp} A}|\gamma \lambda-1|=\max (|\gamma m-1|,|\gamma M-1|)
$$

is minimized. A little thought shows that this is the case iff $|\gamma m-1|=|\gamma M-1|$, i.e. 1 is the midpoint of the interval $[\gamma m, \gamma M]$ so that $\gamma=\frac{2}{M+m}$. In this case,

$$
\|I-\gamma A\|=1-\gamma m=1-\frac{2 m}{M+m}=\frac{M-m}{M+m}=\frac{\kappa-1}{\kappa+1}=1-\frac{2}{\kappa+1}<1 .
$$

Now, by Neumann series, for every $n \in \mathbb{N}_{0}$,

$$
A^{-1}=\gamma(\gamma A)^{-1}=\gamma \sum_{j=0}^{\infty}(I-\gamma A)^{j}=\underbrace{\sum_{j=0}^{n}(I-\gamma A)^{j}}_{=: B_{n}}+\underbrace{\gamma \sum_{j=n+1}^{\infty}(I-\gamma A)^{j}}_{=: C_{n}}
$$

holds with $B_{n} \in \mathrm{BO}_{n k}$ and

$$
\left\|C_{n}\right\| \leq|\gamma| \sum_{j=n+1}^{\infty}\|I-\gamma A\|^{j}=\frac{2}{M+m} \frac{\left(\frac{M-m}{M+m}\right)^{n+1}}{1-\frac{M-m}{M+m}}=\frac{1}{m}\left(\frac{M-m}{M+m}\right)^{n+1}=\frac{1}{m}\left(\frac{\kappa-1}{\kappa+1}\right)^{n+1},
$$

which finishes the proof. 
We now pass to the non-self-adjoint case - but still banded.

Proposition 2.2 Let $A \in \mathrm{BO}_{k}$ for some $k \in \mathbb{N}_{0}$ be invertible and again put $M:=\|A\|, m:=1 /\left\|A^{-1}\right\|$ and $\kappa:=M / m=\|A\|\left\|A^{-1}\right\|$. Then, for every $n \in \mathbb{N}_{0}$, it holds that

$$
\operatorname{dist}\left(A^{-1}, \mathrm{BO}_{3 n k}\right) \leq \frac{M}{m^{2}}\left(\frac{M^{2}-m^{2}}{M^{2}+m^{2}}\right)^{n+1}=\frac{\kappa^{2}}{M}\left(\frac{\kappa^{2}-1}{\kappa^{2}+1}\right)^{n+1}
$$

where an approximation of $A^{-1}$ in $\mathrm{BO}_{3 n k}$ with this accuracy is given in (9) below. In particular, $A^{-1} \in$ $\mathrm{BDO}$ since the right-hand side of (8) goes to zero as $n \rightarrow \infty$.

Proof. The idea is to write $A^{-1}=B^{-1} A^{*}$, where $B:=A^{*} A \in \mathrm{BO}_{2 k}$ is clearly self-adjoint positive definite, and to approximate $B^{-1}$ as in the previous lemma. When we apply Lemma 2.1 to $B$ (in place of $A$ ), note that

$$
\begin{aligned}
M_{B} & :=\|B\|=\left\|A^{*} A\right\|=\|A\|^{2}=M^{2} \\
m_{B} & :=1 /\left\|B^{-1}\right\|=1 /\left\|A^{-1}\left(A^{*}\right)^{-1}\right\|=1 /\left\|A^{-1}\left(A^{-1}\right)^{*}\right\|=1 /\left\|A^{-1}\right\|^{2}=m^{2} \text { and } \\
\kappa_{B} & :=M_{B} / m_{B}=M^{2} / m^{2}=\kappa^{2} .
\end{aligned}
$$

Now for every $n \in \mathbb{N}_{0}$, in analogy to (6), we can write $B^{-1}=B_{n}+C_{n}$ with $B_{n} \in \mathrm{BO}_{2 n k}$ and $\left\|C_{n}\right\|$ bounded as in (7), so that $A^{-1}=B^{-1} A^{*}=B_{n} A^{*}+C_{n} A^{*}$ with

$$
B_{n} A^{*}=\gamma_{B} \sum_{j=0}^{n}\left(I-\gamma_{B} B\right)^{j} A^{*}=\frac{2}{M^{2}+m^{2}} \sum_{j=0}^{n}\left(I-\frac{2 A^{*} A}{M^{2}+m^{2}}\right)^{j} A^{*} \in \mathrm{BO}_{3 n k}
$$

and

$$
\left\|C_{n} A^{*}\right\| \leq \frac{1}{m_{B}}\left(\frac{M_{B}-m_{B}}{M_{B}+m_{B}}\right)^{n+1} M=\frac{M}{m^{2}}\left(\frac{M^{2}-m^{2}}{M^{2}+m^{2}}\right)^{n+1}=\frac{\kappa^{2}}{M}\left(\frac{\kappa^{2}-1}{\kappa^{2}+1}\right)^{n+1},
$$

which proves the result.

Finally, we pass to the most general case, $A \in$ BDO.

Theorem 2.3 Let $A \in \mathrm{BDO}$ be invertible, put $\delta_{k}:=\operatorname{dist}\left(A, \mathrm{BO}_{k}\right)$ for $k=0,1,2, \ldots$ and again let $M:=\|A\|, m:=1 /\left\|A^{-1}\right\|$ and $\kappa:=M / m=\|A\|\left\|A^{-1}\right\|$ as well as $\alpha_{k}:=m /\left(m-2 \delta_{k}\right)$. Note that, by (3), $\delta_{k} \rightarrow 0$ and $\alpha_{k} \rightarrow 1$ as $k \rightarrow \infty$. Then, for all $n \in \mathbb{N}_{0}$ and all sufficiently large $k \in \mathbb{N}_{0}$ (such that $\left.\delta_{k}<m / 2\right)$, it holds that

$$
\begin{aligned}
\operatorname{dist}\left(A^{-1}, \mathrm{BO}_{3 n k}\right) & \leq \frac{\alpha_{k}}{m^{2}}\left(2 \delta_{k}+\alpha_{k}\left(M+2 \delta_{k}\right)\left(\frac{\left(M+2 \delta_{k}\right)^{2}-\left(m / \alpha_{k}\right)^{2}}{\left(M-2 \delta_{k}\right)^{2}+\left(m / \alpha_{k}\right)^{2}}\right)^{n+1}\right) \\
& =\frac{2 \delta_{k} \alpha_{k}}{m^{2}}+\frac{\left(\kappa_{k}^{+}\right)^{2}}{M+2 \delta_{k}}\left(\frac{\left(\kappa_{k}^{+}\right)^{2}-1}{\left(\kappa_{k}^{-}\right)^{2}+1}\right)^{n+1}
\end{aligned}
$$

with $\kappa_{k}^{ \pm}$defined by (11), respectively. In particular, $A^{-1} \in \mathrm{BDO}$.

Proof. For every $k \in \mathbb{N}_{0}$ pick an $A_{k} \in \mathrm{BO}_{k}$ with $\left\|A-A_{k}\right\| \leq 2 \delta_{k}$. Then $A_{k} \rightrightarrows A$ (norm convergence in $\mathrm{BL})$ as $k \rightarrow \infty$ since $\delta_{k} \rightarrow 0$. Since $A$ is invertible we know that $A_{k}$ is invertible for sufficiently large $k$; precisely, take $k_{0} \in \mathbb{N}_{0}$ big enough that $\delta_{k}<m / 2$ for all $k>k_{0}$ so that $\left\|A^{-1}\left(A-A_{k}\right)\right\| \leq 2 \delta_{k} / m<1$. Then $A_{k}=A\left(I-A^{-1}\left(A-A_{k}\right)\right)$ is invertible with

$$
\left\|A_{k}^{-1}\right\|=\left\|\sum_{j=0}^{\infty}\left(A^{-1}\left(A-A_{k}\right)\right)^{j} A^{-1}\right\| \leq \sum_{j=0}^{\infty}\left(\frac{2 \delta_{k}}{m}\right)^{j}\left\|A^{-1}\right\|=\frac{1}{1-\frac{2 \delta_{k}}{m}}\left\|A^{-1}\right\|=\alpha_{k}\left\|A^{-1}\right\|
$$


and hence

$$
\left\|A_{k}^{-1}-A^{-1}\right\|=\left\|A^{-1}\left(A-A_{k}\right) A_{k}^{-1}\right\| \leq\left\|A^{-1}\right\|\left\|A-A_{k}\right\|\left\|A_{k}^{-1}\right\| \leq \frac{1}{m} 2 \delta_{k} \frac{\alpha_{k}}{m}=\frac{2 \delta_{k} \alpha_{k}}{m^{2}}
$$

for all $k>k_{0}$ so that also $A_{k}^{-1} \rightrightarrows A^{-1}$ as $k \rightarrow \infty$. At this point it is clear that $A^{-1} \in \mathrm{BDO}$ since $A_{k}^{-1} \in \mathrm{BDO}$ by Proposition 2.2 and since BDO is closed.

For an explicit approximation of $A^{-1}$ by banded matrices, it remains to look at banded approximations of $A_{k}^{-1}$ and to use (10). Therefore, for every $k>k_{0}$, let $B_{1}^{(k)}, B_{2}^{(k)}, \ldots$ be the banded approximations of $A_{k}^{-1}$ from Proposition 2.2, where $B_{n}^{(k)} \in \mathrm{BO}_{3 n k}$ and $\left\|A_{k}^{-1}-B_{n}^{(k)}\right\|$ is bounded by the right-hand side of (8) with $M, m$ and $\kappa$ replaced by $M_{k}:=\left\|A_{k}\right\|, m_{k}:=1 /\left\|A_{k}^{-1}\right\|$ and $\kappa_{k}:=M_{k} / m_{k}$, respectively. Finally, put

$$
\kappa_{k}^{ \pm}:=\frac{M \pm 2 \delta_{k}}{m / \alpha_{k}}=\alpha_{k}\left(\kappa \pm \frac{2 \delta_{k}}{m}\right)
$$

for every $k \in \mathbb{N}_{0}$. From $\left\|A-A_{k}\right\| \leq 2 \delta_{k}$ and $\left\|A_{k}^{-1}\right\| \leq \alpha_{k}\left\|A^{-1}\right\|$ we get $M-2 \delta_{k} \leq M_{k} \leq M+2 \delta_{k}$ and $m_{k} \geq m / \alpha_{k}$. Hence

$$
\begin{aligned}
\left\|A_{k}^{-1}-B_{n}^{(k)}\right\| & \leq \frac{M_{k}}{m_{k}^{2}}\left(\frac{M_{k}^{2}-m_{k}^{2}}{M_{k}^{2}+m_{k}^{2}}\right)^{n+1} \leq \frac{M+2 \delta_{k}}{\left(m / \alpha_{k}\right)^{2}}\left(\frac{\left(M+2 \delta_{k}\right)^{2}-\left(m / \alpha_{k}\right)^{2}}{\left(M-2 \delta_{k}\right)^{2}+\left(m / \alpha_{k}\right)^{2}}\right)^{n+1} \\
& =\frac{\left(\kappa_{k}^{+}\right)^{2}}{M+2 \delta_{k}}\left(\frac{\left(\kappa_{k}^{+}\right)^{2}-1}{\left(\kappa_{k}^{-}\right)^{2}+1}\right)^{n+1}=\frac{\alpha_{k}^{2}\left(\kappa+\frac{2 \delta_{k}}{m}\right)^{2}}{M+2 \delta_{k}}\left(\frac{\alpha_{k}^{2}\left(\kappa+\frac{2 \delta_{k}}{m}\right)^{2}-1}{\alpha_{k}^{2}\left(\kappa-\frac{2 \delta_{k}}{m}\right)^{2}+1}\right)^{n+1} .
\end{aligned}
$$

Bounding $\left\|A^{-1}-B_{n}^{(k)}\right\| \leq\left\|A^{-1}-A_{k}^{-1}\right\|+\left\|A_{k}^{-1}-B_{n}^{(k)}\right\|$ by (10)+(12) completes the proof.

\section{The Wiener algebra $\mathcal{W}$ is inverse closed}

The term 'Wiener algebra' is commonly used for the set $W(\mathbb{T})$ of all functions $f(t)=\sum_{n \in \mathbb{Z}} f_{n} t^{n}$ on the unit circle $\mathbb{T}$ whose sequence of Fourier coefficients $\hat{f}=\left(f_{n}\right)_{n \in \mathbb{Z}}$ is in $\ell^{1}$ (two-sided infinite), equipped with pointwise addition and multiplication and with the norm $\llbracket f \rrbracket:=\sum\left|f_{n}\right|$. Norbert Wiener's famous theorem says that if $f \in W(\mathbb{T})$ is invertible as a continuous function, i.e. $f$ vanishes nowhere on $\mathbb{T}$, then $f^{-1}=1 / f$ is in $W(\mathbb{T})$ as well, showing that $W(\mathbb{T})$ is inverse closed.

In fact, Wiener's theorem is a special case of our Theorem 3.1 saying that 'our' Wiener algebra $\mathcal{W}$ is inverse closed! It follows if we apply Theorem 3.1 to a two-sided infinite matrix with constant diagonals. To see this take $\mathcal{I}=\mathbb{Z}$ and associate with every function $f \in W(\mathbb{T})$ the so-called Laurent matrix

$$
L(f)=\left(f_{i-j}\right)_{i, j \in \mathbb{Z}}=\left(\begin{array}{ccccc}
\ddots & \ddots & \ddots & \ddots & \ddots \\
\ddots & f_{0} & f_{-1} & f_{-2} & \ddots \\
\ddots & f_{1} & f_{0} & f_{-1} & \ddots \\
\ddots & f_{2} & f_{1} & f_{0} & \ddots \\
\ddots & \ddots & \ddots & \ddots & \ddots
\end{array}\right)
$$

which is sometimes also (wrongly) called a 'two-sided infinite Toeplitz matrix'. Then, clearly,

$$
L(f) \in \mathcal{W} \quad \text { and } \quad \llbracket L(f) \rrbracket=\sum_{n \in \mathbb{Z}}\left|f_{n}\right|=\llbracket f \rrbracket .
$$

Now for $g \in L^{2}(\mathbb{T})$, let $\hat{g}=\left(g_{n}\right)_{n \in \mathbb{Z}} \in \ell^{2}$ denote its sequence of Fourier coefficients and note that $L(f) \hat{g}=\hat{f} * \hat{g}=\widehat{f g}$ acts as the operator of convolution by the sequence $\hat{f}=\left(f_{n}\right)$. In other words, if $F: L^{2}(\mathbb{T}) \rightarrow \ell^{2}$ is the Fourier transform $g \mapsto \hat{g}=\left(g_{n}\right)$ then

$$
L(f)=F M(f) F^{-1}
$$


with $M(f): L^{2}(\mathbb{T}) \rightarrow L^{2}(\mathbb{T})$ denoting the multiplication operator $g \mapsto f g$. The latter formula shows that $L(f)$ is invertible on $\ell^{2}$ iff $M(f)$ is invertible on $L^{2}(\mathbb{T})$ which is clearly the case iff $f$ has no zeros on $\mathbb{T}$. In this case $(L(f))^{-1}=F M\left(f^{-1}\right) F^{-1}=L\left(f^{-1}\right)$ holds, and Wiener's statement, $f^{-1} \in W(\mathbb{T})$, is equivalent to the inverse Laurent matrix $(L(f))^{-1}=L\left(f^{-1}\right)$ being in $\mathcal{W}$. Our Theorem 3.1 however says much more: For all matrices $A \in \mathcal{W}$, not just for those with constant diagonals, the inverse $A^{-1}$, if it exists, is in $\mathcal{W}$.

Theorem 3.1 If $A \in \mathcal{W}$ is invertible then $A^{-1} \in \mathcal{W}$.

Proof. a) As in $\S 2$, we start with the case $A \in \mathrm{BO}$, say $A \in \mathrm{BO}_{k}$ for some $k \in \mathbb{N}_{0}$. Then, by (8),

$$
\operatorname{dist}\left(A^{-1}, \mathrm{BO}_{3 n k}\right) \leq r_{n}:=\frac{\kappa^{2}}{M}\left(\frac{\kappa^{2}-1}{\kappa^{2}+1}\right)^{n+1}
$$

for every $n \in \mathbb{N}_{0}$, where $M=\|A\|, m=1 /\left\|A^{-1}\right\|$ and $\kappa=M / m=\|A\|\left\|A^{-1}\right\|$. Now, for every $j \in \mathbb{Z}$, let $d_{j}$ denote the supremum norm of the $j$-th diagonal of $A^{-1}$. By the previous inequality and (4) we get that

$$
\begin{aligned}
& d_{j} \leq r_{0} \quad \text { for } \quad j \in\{ \pm 1, \ldots, \pm 3 k\}, \\
& d_{j} \leq r_{1} \quad \text { for } \quad j \in\{ \pm(3 k+1), \ldots, \pm 6 k\} \text {, } \\
& d_{j} \leq r_{2} \quad \text { for } \quad j \in\{ \pm(6 k+1), \ldots, \pm 9 k\} \text {, }
\end{aligned}
$$

Summing up we have

$$
\llbracket A^{-1} \rrbracket=\sum_{j \in \mathbb{Z}} d_{j} \leq d_{0}+6 k r_{0}+6 k r_{1}+6 k r_{2}+\ldots=d_{0}+6 k\left(r_{0}+r_{1}+r_{2}+\ldots\right)<\infty
$$

since $r_{n}$ decays exponentially. So $A^{-1} \in \mathcal{W}$ if $A \in \mathrm{BO}$.

b) Now let $A \in \mathcal{W}$ be invertible and take $A_{1}, A_{2}, \ldots \in \mathrm{BO}$ such that $\llbracket A-A_{i} \rrbracket \rightarrow 0$ as $i \rightarrow \infty$. Since $(\mathcal{W}, \llbracket \cdot \rrbracket)$ is a Banach algebra we know that for sufficiently large $i$ also $A_{i}$ is invertible and $\llbracket A^{-1}-A_{i}^{-1} \rrbracket \rightarrow 0$ as $i \rightarrow \infty$. Together with part a) and the closedness of $(\mathcal{W}, \llbracket \cdot \rrbracket)$ this proves the theorem.

Note that one corollary of Theorem 3.1 is that if, for some fixed $p \in[1, \infty]$, an operator $A: \ell^{p} \rightarrow \ell^{p}$ with matrix representation in $\mathcal{W}$ is invertible then its inverse is again given by a matrix in $\mathcal{W}$ and $A^{-1}$ therefore (see (2)) acts boundedly on all spaces $\ell^{p}$ with $p \in[1, \infty]$. So invertibility and spectrum of such operators $A$ do not depend on the particular choice of $p$. In [15] it is shown how this result can be used to prove that even the property of being a Fredholm operator (including the value of the Fredholm index) and hence the essential spectrum of $A$ does not depend on $p \in[1, \infty]$ if $A \in \mathcal{W}$.

\section{Some generalizations}

\subsection{Generalized banding}

It is easy to see that our results generalize well beyond BDO and $\mathcal{W}$. To see what we mean, let $\varrho$ be a metric on $\mathcal{I}$ and define the set $\mathrm{BO}^{\varrho}$ of $\varrho$-generalized banded operators as the set of all $A=\left(a_{i j}\right)_{i, j \in \mathcal{I}} \in \mathrm{BL}$ with $a_{i j}=0$ for all $i, j \in \mathcal{I}$ with $\varrho(i, j)>k$, for some fixed $k$. It is easy to see that $\mathrm{BO}^{\varrho}$ is also closed under addition and multiplication and taking adjoints. Examples of interesting metrics $\varrho$ other than $\varrho(i, j)=|i-j|$ are obtained by taking a sequence $\left(\mathbf{x}_{i}\right)_{i \in \mathcal{I}}$ of pairwise different elements from another metric space $(X, d)$ (e.g. $X=\mathbb{R}^{n}$ with the Euclidean norm) and putting

$$
\varrho(i, j):=d\left(\mathbf{x}_{i}, \mathbf{x}_{j}\right), \quad i, j \in \mathcal{I} .
$$

These generalizations are interesting in statistics as we shall see in Section 6. If we let $\mathrm{BDO}^{\varrho}$ be the closure of $\mathrm{BO}^{\varrho}$ in $\mathrm{BL}\left(\ell^{2}\right)$, Lemma 2.1, Proposition 2.2 and Theorem 2.3 go over verbatim to $\mathrm{BO}^{\varrho}$ and $\mathrm{BDO}^{\varrho}$. 
If we now define the generalized Wiener norm by

$$
\llbracket A \rrbracket_{\varrho}:=\sum_{k \in \mathbb{Z}} d_{k}^{(\varrho)}
$$

where $d_{k}^{(\varrho)}=\sup \left\{\left|a_{i j}\right|: i, j \in \mathcal{I}, \varrho(i, j)=k\right\}$, we can obtain an exact generalization of Theorem 3.1. An obvious application of this result is the generalization of Wiener's theorem to analytic functions

$$
f: \mathbf{t} \mapsto \sum_{\mathbf{n} \in \mathbb{Z}^{p}} f_{\mathbf{n}} \mathbf{t}^{\mathbf{n}}
$$

of several variables, where $\mathbf{t}=\left(t_{1}, \ldots, t_{p}\right) \in \mathbb{T}^{p}, \mathbf{n}=\left(n_{1}, \ldots, n_{p}\right) \in \mathbb{Z}^{p}$ and $\mathbf{t}^{\mathbf{n}}=\prod_{j=1}^{p} t_{j}^{n_{j}}$.

\subsection{Banding up to a permutation}

Let $\pi$ be a permutation, that is, a 1-1 mapping of $\mathcal{I}$ onto itself which is clearly representable as an operator $\left(x_{i}\right)_{i \in \mathcal{I}} \mapsto\left(x_{\pi(i)}\right)_{i \in \mathcal{I}}$ of norm 1 from $\ell^{2}$ to $\ell^{2}$, which we also denote by $\pi$. Define

$$
\mathrm{BO}^{(\pi)}=\left\{A: \pi A \pi^{*} \in B O\right\}
$$

where $\pi^{*}$ is the adjoint/inverse of $\pi \cdot \mathrm{BO}^{(\pi)}$ is closed under addition and multiplication but not under taking adjoints since $A \in \mathrm{BO}^{(\pi)} \Longrightarrow A^{*} \in \mathrm{BO}^{\left(\pi^{*}\right)}$. So we can't expect a generalization of Theorem 2.3. We can, however, generalize Lemma 2.1 and obtain a special case of Theorem 2.3. Specifically, let

$$
\mathrm{HBO}^{(\pi)}:=\left\{A \in \mathrm{BO}^{(\pi)}: A \text { is hermitian positive definite }\right\}
$$

and $\operatorname{HBDO}^{(\pi)}$ be its closure. Then Theorem 2.3 generalizes to $\mathrm{HBDO}^{(\pi)}$ if we replace $B_{3 n k}^{(\pi)}$ by $B_{n k}^{(\pi)}($ with obvious notation changes).

We can also in an obvious way obtain the same conclusions for generalized banding. More important from a statistical point of view is the following generalization. Let

$$
\mathrm{HBO}^{\text {PERM }}:=\bigcup_{\pi} \mathrm{HBO}^{(\pi)} .
$$

Then HBO $^{\text {PERM }}$ is only closed for addition, scalar multiplication, and taking powers. However, an examination of the proof of Theorem 2.3 shows that these properties are sufficient to arrive at the same generalization for $\mathrm{HBO}^{\text {PERM }}$ and its closure $\mathrm{HBDO}^{\text {PERM }}$ as we did for $\mathrm{HBO}^{(\pi)}$ and its closure. Again, everything carries over verbatim to generalized banding. These results, particularly the last, are of interest in statistics since, for reasons to become apparent, it is desirable to define classes of covariance matrices such that matrices and their inverses necessarily obey the same definition of sparseness.

\section{Applications to probability theory}

\subsection{The closures of banded self-adjoint positive definite operators and Gaus- sian processes}

A Gaussian process is a sequence of random variables, $\left\{X_{j}: j \in \mathbb{Z}\right\}$, on a probability space whose finite dimensional joint distributions are Gaussian. Without loss of generality, we take $E X_{j}=0$ for all $j$, so that the joint distributions are determined by the matrices

$$
\Sigma_{m, n}:=E \mathbf{X}_{m}^{n}\left[\mathbf{X}_{m}^{n}\right]^{T}=\left[E X_{i} X_{j}\right]_{i, j=m}^{n}, \quad m, n \in \mathbb{Z},
$$

where we put $\mathbf{X}_{m}^{n}:=\left(X_{m}, \ldots, X_{n}\right)^{T}$. We extend these notations to $m=-\infty$ and $n=\infty$ by introducing the two-sided infinite vector $\mathbf{X}_{-\infty}^{\infty}:=\left(\ldots, X_{-1}, X_{0}, X_{1}, \ldots\right)^{T}$ and matrix

$$
\Sigma:=E \mathbf{X}_{-\infty}^{\infty}\left[\mathbf{X}_{-\infty}^{\infty}\right]^{T}=\left[E X_{i} X_{j}\right]_{i, j=-\infty}^{\infty}
$$


For the rest of our discussion we assume that the infinite matrix $\Sigma$ acts as a bounded operator from $\ell^{2}$ to $\ell^{2}$. A regular process is one for which

$$
E \in \mathcal{B}_{-\infty}:=\bigcap_{t=-\infty}^{\infty} \mathcal{B}_{-\infty}^{t} \quad \Longrightarrow \quad P(E) \in\{0,1\}
$$

where $\mathcal{B}_{m}^{n}$ is the $\sigma$ field generated by $\mathbf{X}_{m}^{n}$ or equivalently

$$
\lim _{p \rightarrow \infty} \sup \left\{|P(A B)-P(A) P(B)|: B \in \mathcal{B}_{-\infty}^{p}\right\}=0
$$

for all $A \in \mathcal{B}_{-\infty}$ and all $p$. A strongly mixing process is one for which

$$
\lim _{p \rightarrow \infty} \beta(p)=0, \quad \text { where } \quad \beta(p)=\sup \left\{|P(A B)-P(A) P(B)|: A \in \mathcal{B}_{-\infty}^{m}, B \in \mathcal{B}_{m+p}^{\infty}, m \in \mathbb{Z}\right\} .
$$

Let $P_{m, n}$ be the joint distribution of $\mathbf{X}_{m}^{n}$, the probability measure induced on $\mathcal{B}_{m}^{n}$ by $P$. Let $P_{m}^{m+n}$ be the regular conditional probability measure on $\mathcal{B}_{m+p}^{\infty}$ given $\mathcal{B}_{-\infty}^{m}$. If $\|\mu\|_{T V}$ denotes the total variation of a signed measure $\mu$, let

$$
\beta(m, p):=E\left\|P_{m}^{m+p}-P_{m+p, \infty}\right\|_{T V} .
$$

A beta mixing (or absolutely regular) process is one such that

$$
\lim _{p \rightarrow \infty} \sup _{m} \beta(m, p)=0 .
$$

As noted in [5], beta mixing implies strong mixing. The converse is not true as Example 5.3 below shows. On the other hand, let $Q_{m, n}$ be the probability distribution induced by $P$ on the $\sigma$ field generated by $\mathcal{B}_{-\infty}^{m}$ and $\mathcal{B}_{n}^{\infty}$. Let $\bar{Q}_{m, n}$ be the product probability on the same $\sigma$ field with marginals $P_{-\infty, m}, P_{n, \infty}$. Then Lemma 2 of $[5$, p. 118] states

$$
\beta(m, p)=\frac{1}{2}\left\|Q_{m, m+p}-\bar{Q}_{m, m+p}\right\|_{T V} .
$$

A mean 0 process is linearly regular if

$$
E\left(X_{p+m+1} \mid X_{-\infty}^{m}\right) \rightarrow 0
$$

as $p \rightarrow \infty$, uniformly in $m$. A detailed discussion of these concepts is in Ibragimov and Rozanov [5] primarily in the context of stationary processes.

For Gaussian processes, linear regularity and regularity are equivalent, see [5, p.112]. We connect with our previous results via

Theorem 5.1 If $\Sigma$ has a bounded inverse and belongs to $\mathrm{BDO}$ then $\mathbf{X}_{-\infty}^{\infty}$ is regular, and so is the process corresponding to $\Sigma^{-1}$.

Proof. Recall $\mathbf{X}_{a}^{b}=\left(X_{a}, X_{a+1}, \ldots, X_{b}\right)^{T}$ and let

$$
\Sigma(a, b)=E \mathbf{X}_{a}^{b}\left[\mathbf{X}_{a}^{b}\right]^{T}=\left[E X_{i} X_{j}\right]_{i, j=a}^{b}
$$

denote the $(b-a+1) \times(b-a+1)$ diagonal block of $\Sigma$. Moreover let

$$
\boldsymbol{\sigma}(a, b, c)=E \mathbf{X}_{a}^{b} X_{c} .
$$

Then

$$
\begin{aligned}
E\left(X_{m+p+1} \mid \mathbf{X}_{-m}^{n}\right) & =\left[\mathbf{X}_{-m}^{n}\right]^{T} \Sigma^{-1}(-m, n) \boldsymbol{\sigma}(-m, n, n+p+1) \\
E\left[E\left(X_{n+p+1} \mid \mathbf{X}_{-m}^{n}\right)\right]^{2} & =\boldsymbol{\sigma}^{T}(-m, n, n+p+1) \Sigma^{-1}(-m, n) \boldsymbol{\sigma}(-m, n, n+p+1) .
\end{aligned}
$$


Since $\Sigma$ is a member of BDO we can find $B_{\varepsilon}$ banded of width $k(\varepsilon)$ such that $\left\|B_{\varepsilon}-\Sigma\right\| \leq \varepsilon$. Let $\left(X_{-m}(\varepsilon), \ldots, X_{n+p+1}(\varepsilon), \ldots\right)$ be a Gaussian process with covariance operator $B_{\varepsilon}$, (a moving average process). Then,

$$
\begin{aligned}
\left\|B_{\varepsilon}(-m, n)-\Sigma(-m, n)\right\| & \leq \varepsilon \\
\left|\boldsymbol{\sigma}_{\varepsilon}(-m, n, n+p+1)-\boldsymbol{\sigma}(-m, n, n+p+1)\right| & \leq \varepsilon
\end{aligned}
$$

where $\boldsymbol{\sigma}_{\varepsilon}(a, b, c)=E \mathbf{X}_{a}^{b} X_{c}(\varepsilon)$ and $|\cdot|$ is the Euclidean norm. By construction,

$$
\boldsymbol{\sigma}_{\varepsilon}(-m, n, n+p+1)=\mathbf{0} \quad \text { if } \quad p \geq k(\varepsilon) .
$$

Then, from (16) and (17),

$$
\left|\boldsymbol{\sigma}^{T}(-m, n, n+p+1) \Sigma^{-1}(-m, n) \boldsymbol{\sigma}(-m, n, n+p+1)\right| \leq \varepsilon\left\|\Sigma^{-1}\right\| \varepsilon
$$

for all $p \geq k(\varepsilon)$. The main result follows from (15) and (18). The corresponding statement for $\Sigma^{-1}$ is a consequence of Theorem 2.3.

\subsection{Beta mixing and Frobenius closure of banded operators}

In general, $\Sigma \in$ BDO having a bounded inverse does not imply beta mixing (see Example 5.3 below). But below we prove that beta mixing is equivalent to a condition on the off-diagonal decay of $\Sigma$ which can be related to the closure of BO in a type of Frobenius norm.

In the negative, there are results of Kolmogorov and Rozanov [8] for symmetric positive definite Toeplitz matrices $\Sigma$, i.e. one-sided infinite versions of (13) with $f_{n}=f_{-n}$, showing that strong (and hence beta) mixing does not hold if the associated symbol $f: t \in \mathbb{T} \mapsto \sum_{n \in \mathbb{Z}} f_{n} t^{n} \in \mathbb{R}$ has discontinuities of the first kind. (Recall from Section 3 that $\Sigma$ is: bounded iff $f \in L^{\infty}(\mathbb{T})$, invertible iff $1 / f \in L^{\infty}(\mathbb{T})$, and it is in BDO iff $f$ is continuous.)

On the positive side, Ibragimov and Rozanov [5, p.129] establish

Theorem 5.2 If $f$ is the symbol of a (one-or two-sided infinite) Toeplitz matrix $\Sigma$ corresponding to a stationary Gaussian process $X$ and

$$
f\left(e^{i x}\right)=\left|P\left(e^{i x}\right)\right|^{2} a(x), \quad x \in(-\pi, \pi)
$$

where $P$ is a polynomial with zeros, if any, only on the unit circle and $\log a(\cdot)$ belongs to the Sobolev space $W^{\frac{1}{2}, 2}$ then $X$ is beta mixing and conversely.

Recall that

$$
W^{s, 2}=\left\{b(x)=\sum_{k \in \mathbb{Z}} a_{k} e^{i k x}: \sum_{k \in \mathbb{Z}}|k|^{2 s}\left|a_{k}\right|^{2}<\infty\right\} .
$$

Note that if $f$ is bounded above and away from zero then one can take $P \equiv 1$, and the condition $\log a(\cdot) \in W^{\frac{1}{2}, 2}$ is equivalent to $a \in W^{\frac{1}{2}, 2}$ and $a$ bounded away from zero. To see the latter, note that $W^{\frac{1}{2}, 2}$ can be equivalently characterized by the Sobolev-Slobodeckij norm (e.g. [18]), in which it becomes clear that with $f$ also powers of $f$ and hence, by closedness, also $\log f$ (if $f$ is bounded above and away from zero) and $\exp f$ are in $W^{\frac{1}{2}, 2}$.

Here is an example of a strong but not beta mixing stationary process.

Example 5.3 For $k \in \mathbb{Z}$, let $a_{k}=1 / \sqrt{|k|}$ if $|k| \in\left\{1^{4}, 2^{4}, 3^{4}, \ldots\right\}$, and $a_{k}=0$ otherwise. Now look at the symbol function $f(t)=\sum_{k \in \mathbb{Z}} a_{k} t^{k}$ defined on the unit circle $\mathbb{T}$. Because of $a_{k}=a_{-k}$, the symbol $f$ is real-valued. One moreover has

$$
\sum_{k \in \mathbb{Z}}\left|a_{k}\right|=2 \sum_{m \in \mathbb{N}} \frac{1}{\sqrt{m^{4}}}=2 \sum_{m \in \mathbb{N}} \frac{1}{m^{2}}=\frac{\pi^{2}}{3}<\infty,
$$


so that $f$ is in the Wiener class $W(\mathbb{T})$ that we discuss at the beginning of Section 3 , and hence $f$ is continuous. In particular, $f$ is bounded with

$$
|f(t)|=\left|\sum_{k \in \mathbb{Z}} a_{k} t^{k}\right| \leq \sum_{k \in \mathbb{Z}}\left|a_{k}\right|=\frac{\pi^{2}}{3}<4, \quad t \in \mathbb{T},
$$

so that $f(t) \in\left(-\frac{\pi^{2}}{3}, \frac{\pi^{2}}{3}\right) \subset(-4,4)$ for all $t \in \mathbb{T}$. However, $f$ is not in the Sobolev space $W^{\frac{1}{2}, 2}$ since

$$
\sum_{k \in \mathbb{Z}}|k|\left|a_{k}\right|^{2}=2 \sum_{m \in \mathbb{N}} m^{4} \frac{1}{m^{4}}=\infty .
$$

Putting $g(t):=f(t)+4$, we get that the Fourier coefficients $b_{k}$ of $g$ coincide with $a_{k}$, except $b_{0}$ which is 4. Now the range of $g$ is in $\left(4-\frac{\pi^{2}}{3}, 4+\frac{\pi^{2}}{3}\right) \subset(0,8)$, so that $g$ is positive, bounded away from zero, and continuous, whence the associated process with covariance matrix $\Sigma=\left(b_{i-j}\right)_{i, j}$ is strong mixing. But the process is not beta mixing since $g \notin W^{\frac{1}{2}, 2}$.

We now give a generalization of Theorem 5.2 to arbitrary bounded covariance matrices $\Sigma$. Implicitly, the result is essentially in Lemmas $2-5$ of $[5, \S I V .4]$ but we give a full statement and proof here for completeness. We denote the entries of our infinite covariance matrix $\Sigma$ by $\sigma_{i j}=E X_{i} X_{j}$ for $i, j \in \mathbb{Z}$.

Theorem 5.4 Suppose $\Sigma \in \mathrm{BL}$ is invertible and $E \mathbf{X}_{-\infty}^{\infty}=\mathbf{0}$. Then $\mathbf{X}_{-\infty}^{\infty}$ Gaussian is beta mixing iff

$$
\sup _{n \in \mathbb{Z}} \sum_{i=-\infty}^{n} \sum_{j=p+n}^{\infty} \sigma_{i j}^{2} \rightarrow 0 \quad \text { as } \quad p \rightarrow \infty .
$$

A simpler sufficient condition is given by

$$
\gamma(p)<\infty \text { for some } p \geq 1, \quad \text { where } \quad \gamma(p):=\sum_{i=-\infty}^{\infty} \sum_{j=p+i}^{\infty} \sigma_{i j}^{2},
$$

since $(20) \Rightarrow(19)$.

Proof. Note that with $\Sigma$ also $\Sigma^{-1}$ is in BL and put $M:=\max \left(\|\Sigma\|,\left\|\Sigma^{-1}\right\|\right)<\infty$. By (14), to prove the theorem we need only show that (19) is equivalent to

$$
\sup _{m}\left\|Q_{m, m+p}-\bar{Q}_{m, m+p}\right\|_{T V} \rightarrow 0 \quad \text { as } \quad p \rightarrow \infty .
$$

Moreover, it is easy to see that (21) is equivalent to

$$
\sup _{m, n, k}\left\|Q_{m, n, p, k}-\bar{Q}_{m, n, p, k}\right\|_{T V} \rightarrow 0 \quad \text { as } \quad p \rightarrow \infty,
$$

where $Q_{m, n, p, k}$ and $\bar{Q}_{m, n, p, k}$ are the distributions $Q_{m, m+p}$ and $\bar{Q}_{m, m+p}$ restricted to $\mathcal{B}_{m}^{n}, \mathcal{B}_{n+p}^{n+p+k}$.

We consider $\mathbf{X}^{(1)}:=\left(X_{m}, \ldots, X_{n}\right)^{T}, \mathbf{X}^{(2)}:=\left(X_{n+p+1}, \ldots, X_{n+p+k}\right)^{T}$. Let $f$ denote the joint density of $\left(\mathbf{X}^{(1)}, \mathbf{X}^{(2)}\right)$ so that $f$ corresponds to

$$
S=\left(\begin{array}{cc}
\Sigma_{11} & \Sigma_{12} \\
\Sigma_{21} & \Sigma_{22}
\end{array}\right)
$$

the covariance matrix of $\left(\mathbf{X}^{(1)}, \mathbf{X}^{(2)}\right)$ blocked out. Thus,

$$
\mathbf{X}^{(1)} \sim N_{n-m+1}\left(0, \Sigma_{11}\right) \quad \text { and } \quad \mathbf{X}_{2} \sim N_{k}\left(0, \Sigma_{22}\right) .
$$

Let $g$ correspond to

$$
S_{0}:=\left(\begin{array}{cc}
\Sigma_{11} & 0 \\
0 & \Sigma_{22}
\end{array}\right)
$$


the covariance matrix of $\left(\mathbf{X}^{(1)}, \mathbf{X}^{(2)}\right)$ if $\mathbf{X}^{(1)}$ and $\mathbf{X}^{(2)}$ are independent. Let $P_{f}, P_{g}$ be the probability distributions of $\mathbf{X}^{(1)}$ and $\mathbf{X}^{(2)}$ and

$$
\left\|P_{f}-P_{g}\right\|_{T V}:=\frac{1}{2} \int|f-g|
$$

be the variational norm. We suppress the dependence of $f, g$ on $m, p, k$ in what follows. Let

$$
H^{2}\left(P_{f}, P_{g}\right):=2\left(1-\int[f g]^{\frac{1}{2}}\right)=: 2(1-A(f, g))
$$

be the (squared) Hellinger metric. It is well known that

$$
\frac{1}{2} H^{2}\left(P_{f}, P_{g}\right) \leq\left\|P_{f}-P_{g}\right\|_{T V} \leq \sqrt{2} H^{2}\left(P_{f}, P_{g}\right) .
$$

Thus we can replace $\left\|Q_{m, n, p, k}-\bar{Q}_{m, n, p, k}\right\|_{T V}$ by $H^{2}\left(Q_{m, n, p, k}, \bar{Q}_{m, n, p, k}\right)$ in (22).

Our argument will consist of bounding $\frac{1}{2} H^{2}=1-A$ above and below by functions $\underline{a}_{m, n, p, k}$ and $\bar{a}_{m, n, p, k}$ of $\left\{\sigma_{i j}^{2}: m \leq i \leq n, n+p+1 \leq j \leq n+p+k\right\}$ (see (30) below) such that

$$
\sup _{m, n, k} \underline{\bar{a}}_{m, n, p, k} \rightarrow 0 \quad \text { as } \quad p \rightarrow \infty
$$

iff (19) holds. To do this, we have to compute $A(f, g)$.

Note that $\|\cdot\|_{T V}$ and $H^{2}(\cdot, \cdot)$ are invariant under regular linear transformations $\mathbf{X}^{(1)} \rightarrow T_{1} \mathbf{X}^{(1)}$ and $\mathbf{X}^{(2)} \rightarrow T_{2} \mathbf{X}^{(2)}$. For the choice of these matrices $T_{1}$ and $T_{2}$, suppose, by the spectral theorem, that $\Sigma_{j j}=Q_{j}^{T} \Lambda_{j} Q_{j}, j=1,2$, where $Q_{j}$ are orthogonal and $\Lambda_{j}$ are diagonal, and put

$$
T_{1}=\Lambda_{1}^{-\frac{1}{2}} Q_{1} \quad \text { and } \quad T_{2}=\Lambda_{2}^{-\frac{1}{2}} Q_{2} .
$$

Replacing $\left(\mathbf{X}^{(1)}, \mathbf{X}^{(2)}\right)$ by $\left(T_{1} \mathbf{X}^{(1)}, T_{2} \mathbf{X}^{(2)}\right)$ in the above, we get that $\Sigma_{11}=I_{1}$ and $\Sigma_{22}=I_{2}$ are the $(n-m+1) \times(n-m+1)$ and $k \times k$ identity, respectively. We shall establish the theorem in this case and then derive the general case.

If $\Sigma_{11}=I_{1}$ and $\Sigma_{22}=I_{2}$ then, in corresponding block notation,

$$
S^{-1}=\left(\begin{array}{cc}
\Sigma^{11} & \Sigma^{12} \\
\Sigma^{21} & \Sigma^{22}
\end{array}\right)
$$

with

$$
\begin{aligned}
\Sigma^{11} & =\left(I_{1}-\Sigma_{12} \Sigma_{21}\right)^{-1} \\
\Sigma^{22} & =\left(I_{2}-\Sigma_{21} \Sigma_{12}\right)^{-1} \\
\Sigma^{12} & =-\left(I_{1}-\Sigma_{12} \Sigma_{21}\right)^{-1} \Sigma_{12} \\
\Sigma^{21} & =-\left(I_{2}-\Sigma_{21} \Sigma_{12}\right)^{-1} \Sigma_{21}
\end{aligned}
$$

and the determinant of $S$ is equal to

$$
|S|=\left|I_{1}-\Sigma_{12} \Sigma_{21}\right|=\left|I_{2}-\Sigma_{21} \Sigma_{12}\right|
$$

since $\Sigma_{12} \Sigma_{21}$ and $\Sigma_{21} \Sigma_{12}$ have the same nonzero eigenvalues. It holds that

$$
A(f, g)=\int(f g)^{\frac{1}{2}}=|S|^{-\frac{1}{4}}(2 \pi)^{-\frac{n-m+1+k}{2}} \int e^{-\frac{1}{2} \mathbf{x}^{T} \frac{1}{2}\left(S^{-1}+S_{0}^{-1}\right) \mathbf{x}} d \mathbf{x}=|S|^{-\frac{1}{4}}\left|\frac{1}{2}\left(S^{-1}+S_{0}^{-1}\right)\right|^{-\frac{1}{2}}
$$

with $\mathbf{x}=\left(\begin{array}{l}\mathbf{x}_{1} \\ \mathbf{x}_{2}\end{array}\right) \in \mathbb{R}^{m} \times \mathbb{R}^{k}$ and

$$
\begin{aligned}
S^{-1}+S_{0}^{-1} & =\left(\begin{array}{cc}
\Sigma^{11}+I_{1} & \Sigma^{12} \\
\Sigma^{21} & \Sigma^{22}+I_{2}
\end{array}\right) \\
& =\left(\begin{array}{cc}
\left(I_{1}-\Sigma_{12} \Sigma_{21}\right)^{-1}+I_{1} & -\left(I_{1}-\Sigma_{12} \Sigma_{21}\right)^{-1} \Sigma_{12} \\
-\left(I_{2}-\Sigma_{21} \Sigma_{12}\right)^{-1} \Sigma_{21} & \left(I_{2}-\Sigma_{21} \Sigma_{12}\right)^{-1}+I_{2}
\end{array}\right)=:\left(\begin{array}{cc}
M_{11} & M_{12} \\
M_{21} & M_{22}
\end{array}\right) .
\end{aligned}
$$


To compute (25), recall (24), (26) and the standard formula for block determinants

$$
\left|S^{-1}+S_{0}^{-1}\right|=\left|M_{11}\right|\left|M_{22}-M_{21} M_{11}^{-1} M_{12}\right| .
$$

W.l.o.g suppose $n-m+1 \leq k$. Now let $\lambda_{m}, \ldots, \lambda_{n}$ be the eigenvalues of $\Sigma_{12} \Sigma_{21}$. Then $\Sigma_{21} \Sigma_{12}$ has the same $n-m+1$ eigenvalues and the rest are zeros. Let $x$ be an eigenvector of $\Sigma_{21} \Sigma_{12}$ corresponding to $\lambda$. Then $\Sigma_{12} x$ is an eigenvector of $\Sigma_{12} \Sigma_{21}$ corresponding to the same $\lambda$. Consequently, for such an $x$, we have

$$
M_{22} x=\left(\left(I_{2}-\Sigma_{21} \Sigma_{12}\right)^{-1}+I_{2}\right) x=\left((1-\lambda)^{-1}+1\right) x=(1-\lambda)^{-1}(2-\lambda) x
$$

and

$$
\begin{aligned}
M_{21} M_{11}^{-1} M_{12} x & =\left(I_{2}-\Sigma_{21} \Sigma_{12}\right)^{-1} \Sigma_{21}\left(\left(I_{1}-\Sigma_{12} \Sigma_{21}\right)^{-1}+I_{1}\right)^{-1}\left(I_{1}-\Sigma_{12} \Sigma_{21}\right)^{-1} \Sigma_{12} x \\
& =\left(I_{2}-\Sigma_{21} \Sigma_{12}\right)^{-1} \Sigma_{21}\left((1-\lambda)^{-1}+1\right)^{-1}(1-\lambda)^{-1} \Sigma_{12} x \\
& =(1-\lambda)^{-1}\left((1-\lambda)^{-1}+1\right)^{-1}(1-\lambda)^{-1} \lambda x \\
& =(1-\lambda)^{-1}(2-\lambda)^{-1} \lambda x .
\end{aligned}
$$

Taking this together with (26) and (27), we get

$$
\begin{aligned}
\left|\frac{1}{2}\left(S^{-1}+S_{0}^{-1}\right)\right| & =\prod_{j=m}^{n} \frac{1}{2}\left(\frac{1}{1-\lambda_{j}}+1\right) \prod_{j=1}^{k} \frac{1}{2}\left(\frac{2-\lambda_{j}}{1-\lambda_{j}}-\frac{\lambda_{j}}{\left(1-\lambda_{j}\right)\left(2-\lambda_{j}\right)}\right) \\
& =\prod_{j=m}^{n} \frac{1}{4} \frac{2-\lambda_{j}}{1-\lambda_{j}} \frac{\left(2-\lambda_{j}\right)^{2}-\lambda_{j}}{\left(1-\lambda_{j}\right)\left(2-\lambda_{j}\right)}=\prod_{j=m}^{n} \frac{\left(4-\lambda_{j}\right)\left(1-\lambda_{j}\right)}{4\left(1-\lambda_{j}\right)^{2}}=\prod_{j=m}^{n} \frac{1-\frac{\lambda_{j}}{4}}{1-\lambda_{j}}
\end{aligned}
$$

so that, by (24), (25) and (28),

$$
A(f, g)=|S|^{-\frac{1}{4}}\left|\frac{1}{2}\left(S^{-1}+S_{0}^{-1}\right)\right|^{-\frac{1}{2}}=\left(\prod_{j=m}^{n}\left(1-\lambda_{j}\right)\right)^{-\frac{1}{4}}\left(\prod_{j=m}^{n} \frac{1-\frac{\lambda_{j}}{4}}{1-\lambda_{j}}\right)^{-\frac{1}{2}}=\prod_{j=m}^{n} \frac{\left(1-\lambda_{j}\right)^{\frac{1}{4}}}{\left(1-\frac{\lambda_{j}}{4}\right)^{\frac{1}{2}}}
$$

where we recall that $0 \leq \lambda_{j}<1$ for all $j$ since $\Sigma_{12} \Sigma_{21}$ is positive semi-definite and $\left(\Sigma^{11}\right)^{-1}=I_{1}-\Sigma_{12} \Sigma_{21}$ is positive definite.

Now we put

$$
t:=\operatorname{Trace}\left(\Sigma_{12} \Sigma_{21}\right)=\sum_{j=m}^{n} \lambda_{j}
$$

and note that our condition (19) is equivalent to

$$
t=\operatorname{Trace}\left(\Sigma_{12} \Sigma_{21}\right)=\sum_{i=m}^{n}\left[\sum_{j=n+p+1}^{n+p+k} \sigma_{i j} \sigma_{j l}\right]_{i=l}=\sum_{i=m}^{n} \sum_{j=n+p+1}^{n+p+k} \sigma_{i j}^{2} \rightarrow 0 \quad \text { as } \quad p \rightarrow \infty
$$

uniformly in $m, n, k$. The inequalities

$$
\prod_{j=m}^{n}\left(1-\lambda_{j}\right) \geq 1-\sum_{j=m}^{n} \lambda_{j}, \quad 0 \leq \lambda_{m}, \ldots, \lambda_{n} \leq 1
$$

(as can be seen by induction over the number of terms) and

$$
\frac{1-\lambda}{\left(1-\frac{\lambda}{4}\right)^{2}} \leq e^{-\frac{1}{2} \lambda}, \quad \lambda \geq 0
$$

(which is easily checked using basic calculus), together with (29), yield

$$
(1-t)^{\frac{1}{4}} \leq \prod_{j=m}^{n}\left(1-\lambda_{j}\right)^{\frac{1}{4}} \leq A(f, g) \leq e^{-\frac{1}{8} t} \leq 1 .
$$


From (30) we get that $t \rightarrow 0$ implies $A(f, g) \rightarrow 1$. Conversely, by the right half of $(30), A(f, g) \rightarrow 1$ implies $t \rightarrow 0$.

Thus the result is proved if $\Sigma_{11}$ and $\Sigma_{22}$ are the identity.

General case. By the spectral theorem we noted we can find $Q_{1}$ and $Q_{2}$ orthogonal such that

$$
\Sigma_{11}=Q_{1}^{T} \Lambda_{1} Q_{1} \quad \text { and } \quad \Sigma_{22}=Q_{2}^{T} \Lambda_{2} Q_{2},
$$

where $\Lambda_{1}$ and $\Lambda_{2}$ are diagonal. The transformation of $\mathbb{R}^{m+k}$ by $\left(\begin{array}{cc}Q_{1} & 0 \\ 0 & Q_{2}\end{array}\right)$ doesn't change Hellinger or variational distances and sends $\Sigma_{i j} \mapsto \tilde{\Sigma}_{i j}$, where

$$
\tilde{\Sigma}_{11}=\Lambda_{1}, \quad \tilde{\Sigma}_{22}=\Lambda_{2}, \quad \tilde{\Sigma}_{12}=Q_{1} \Sigma_{12} Q_{2}^{T}, \quad \tilde{\Sigma}_{21}=Q_{2} \Sigma_{21} Q_{1}^{T} \quad \text { and hence } \quad \tilde{\Sigma}_{12} \tilde{\Sigma}_{21}=Q_{1} \Sigma_{12} \Sigma_{21} Q_{1}^{T} .
$$

But then

$$
\operatorname{Trace}\left(\Sigma_{12} \Sigma_{21}\right)=\operatorname{Trace}\left(\tilde{\Sigma}_{12} \tilde{\Sigma}_{21}\right)
$$

since eigenvalues are unchanged. Sending

$$
\mathbf{X}^{(1)} \mapsto \Lambda_{1}^{-\frac{1}{2}} Q_{1} \mathbf{X}^{(1)} \quad \text { and } \quad \mathbf{X}^{(2)} \mapsto \Lambda_{2}^{-\frac{1}{2}} Q_{2} \mathbf{X}^{(2)}
$$

again doesn't change Hellinger and TV distances. If the resulting covariance matrices are $\Sigma_{i j}^{*}$ then

$$
\Sigma_{11}^{*}=\Sigma_{22}^{*}=J \quad \text { and } \quad \Sigma_{12}^{*} \Sigma_{21}^{*}=\Lambda_{1}^{-\frac{1}{2}} \tilde{\Sigma}_{12} \Lambda_{2}^{-1} \tilde{\Sigma}_{21} \Lambda_{1}^{-\frac{1}{2}} .
$$

Then

$$
\operatorname{Trace}\left(\Sigma_{12}^{*} \Sigma_{21}^{*}\right)=\sum_{i=m}^{n} \sum_{j=n+p+1}^{n+p+k} \frac{\tilde{\sigma}_{i j}^{2}}{\lambda_{1}^{(i)} \lambda_{2}^{(i)}}
$$

where $\tilde{\Sigma}_{12}=\left(\tilde{\sigma}_{i j}\right)$ and $\lambda_{1}^{(i)}, \lambda_{2}^{(i)}$ are the diagonal elements of $\Lambda_{1}, \Lambda_{2}$. But since

$$
M^{-1} \leq\left\|\Sigma^{-1}\right\|^{-1} \leq \lambda_{1}^{(i)}, \lambda_{2}^{(i)} \leq\|\Sigma\| \leq M, \quad i=m, \ldots, n
$$

it follows that

$$
M^{-2} \operatorname{Trace}\left(\Sigma_{12} \Sigma_{21}\right) \leq \operatorname{Trace}\left(\Sigma_{12}^{*} \Sigma_{21}^{*}\right) \leq M^{2} \operatorname{Trace}\left(\Sigma_{12} \Sigma_{21}\right)
$$

by (31) and (32). Now the theorem follows.

Remark 5.5 If we suppose $\Sigma$ to be a Laurent matrix, i.e. $\Sigma=\left(a_{i-j}\right)_{i, j \in \mathbb{Z}}$ with $a_{k}=a_{-k}$ for all $k$, then it is easy to see that (19) is equivalent to

$$
\sum_{i=1}^{\infty} \sum_{k=p+i}^{\infty} a_{k}^{2} \rightarrow 0, \quad \text { which evidently holds iff } \quad \sum_{i=1}^{\infty} \sum_{k=i+1}^{\infty} a_{k}^{2}<\infty,
$$

i.e. iff

$$
\sum_{k=1}^{\infty} k a_{k}^{2}<\infty
$$

This is the $W^{\frac{1}{2}, 2}$ condition from [5] (see Theorem 5.2).

We now prove a closure under inversion result similar to those of Sections 2 and 3 . Therefore, consider the cone of bounded self-adjoint positive definite operators $\Sigma=\left(\sigma_{i j}\right)$ with bounded inverses and, for $m=0,1,2, \ldots$, denote the subcone of all such operators with

$$
\sum_{|i-j| \geq m} \sigma_{i j}^{2}<\infty
$$


by $F_{m}$. Define on $F_{m}$ an equivalence relation by

$$
\left(\sigma_{i j}\right) \equiv\left(\tau_{i j}\right)
$$

iff $\sigma_{i j} \chi(|i-j| \geq m)=\tau_{i j} \chi(|i-j| \geq m)$, where

$$
\chi(|i-j| \geq m)= \begin{cases}1 & \text { if }|i-j| \geq m \\ 0 & \text { otherwise. }\end{cases}
$$

Let $\Sigma_{0}:=\left(\sigma_{i j} \chi(|i-j| \geq m)\right)$ correspond to such an equivalence class and define

$$
\left\|\Sigma_{0}\right\|_{F_{m}}^{2}:=\sum_{|i-j| \geq m} \sigma_{i j}^{2} .
$$

The quotient cone, which we again denote by $F_{m}$, is closed under convex combination and positive scaling but not under multiplication. However it is closed under Schur multiplication

$$
\Sigma_{0} * \mathcal{T}_{0}:=\left(\sigma_{i j} \tau_{i j} \chi(|i-j| \geq m)\right),
$$

where $\Sigma_{0}, \mathcal{T}_{0}$ and $\Sigma_{0} * \mathcal{T}_{0}$ are equivalence classes. Clearly

$$
\left\|\Sigma_{0} * \mathcal{T}_{0}\right\|_{F_{m}} \leq\left\|\Sigma_{0}\right\|_{F_{m}}\left\|\mathcal{T}_{0}\right\|_{F_{m}} .
$$

Now note that $F_{0} \subset F_{1} \subset \cdots$ and put

$$
F:=\bigcup_{m \geq 0} F_{m},
$$

so that a matrix $\Sigma=\left(\sigma_{i j}\right)$ is in $F$ iff $\|\Sigma\|_{F_{m}}<\infty$ for some $m \geq 0$. For $\Sigma \in \cap_{m \geq 0} F_{m}=F_{0}$, one has that

$$
\sup _{m \geq 0}\|\Sigma\|_{F_{m}}^{2}=\|\Sigma\|_{F_{0}}^{2}=\sum_{i, j} \sigma_{i j}^{2}
$$

is the usual Frobenius norm.

We note the connection to BO. Evidently, $\mathrm{BO}_{m} \subset F_{m+1}$ for all $m \geq 0$ and hence $\mathrm{BO} \subset F$. In fact, $F$ is the closure of $\mathrm{BO}$ in the Frobenius metric $d_{F}(\Sigma, \mathcal{T}):=\|\Sigma-\mathcal{T}\|_{F_{0}}$. To see the latter, note that $F$ is closed under $d_{F}(\cdot, \cdot)$ and take $\Sigma=\left(\sigma_{i j}\right) \in F$, so that $\Sigma \in F_{m_{0}}$, i.e. $\sum_{|i-j| \geq m_{0}} \sigma_{i j}^{2}<\infty$, for some $m_{0} \geq 0$. Then, putting $B_{m}:=\left(\sigma_{i j} \chi(|i-j|<m)\right) \in \mathrm{BO}_{m}$ for $m=0,1, \ldots$, it holds that

$$
d_{F}\left(\Sigma, B_{m}\right)^{2}=\left\|\Sigma-B_{m}\right\|_{F_{0}}^{2}=\left\|\Sigma-B_{m}\right\|_{F_{m}}^{2}=\sum_{|i-j| \geq m} \sigma_{i j}^{2} \rightarrow 0 \quad \text { as } \quad m \rightarrow \infty .
$$

We will see now that, besides BDO and $\mathcal{W}$ (see Theorem 2.3 and 3.1), which are the closures of BO under $\|\cdot\|$ and $\llbracket \cdot \rrbracket$, respectively, also the Frobenius closure $F$ of BO is inverse closed.

Theorem 5.6 F is closed under inversion.

As we have noted in Theorem 5.4, all operators in $F$ are beta mixing so that we conclude that at least on $F$ beta mixing is preserved under inversion.

Proof. Let $\mathbf{Y}_{-\infty}^{\infty}=\left(\ldots, Y_{m}, \ldots, Y_{n}, \ldots\right)^{T}$ have covariance operator $\Sigma^{-1}$ and, following our previous practice from the proof of Theorem 5.4, consider $\mathbf{Y}^{(1)}:=\left(Y_{m}, \ldots, Y_{n}\right)^{T}$ and $\mathbf{Y}^{(2)}:=\left(Y_{n+p+1}, \ldots, Y_{n+p+k}\right)^{T}$ and the corresponding covariance matrices, $\Sigma^{11}, \Sigma^{12}, \Sigma^{21}, \Sigma^{22}$. Following our previous argument we need only check that

$$
\operatorname{Trace}\left(\Sigma^{12} \Sigma^{21}\right) \rightarrow 0
$$

as $p \rightarrow \infty$ uniformly in $m, n, k$. As before we can reduce to the case where $\Sigma^{11}$ and $\Sigma^{22}$ are the identity. Hence, by formula (23),

$$
\Sigma^{12}=-\left(I_{1}-\Sigma_{12} \Sigma_{21}\right)^{-1} \Sigma_{12} \quad \text { and } \quad \Sigma^{21}=\left(\Sigma^{12}\right)^{T}=-\Sigma_{21}\left(I_{1}-\Sigma_{12} \Sigma_{21}\right)^{-1},
$$


so that

$$
\operatorname{Trace}\left(\Sigma^{12} \Sigma^{21}\right)=\operatorname{Trace}\left(\Sigma^{21} \Sigma^{12}\right)=\operatorname{Trace}\left(\Sigma_{21}\left(I_{1}-\Sigma_{12} \Sigma_{21}\right)^{-2} \Sigma_{12}\right) .
$$

Next, note that

$$
\left\|\Sigma_{12} \Sigma_{21}\right\|_{F}^{2}=\sum_{j=m}^{n} \lambda_{j}^{2} \leq\left(\sum_{j=m}^{n} \lambda_{j}\right)^{2}=\left(\operatorname{Trace}\left(\Sigma_{12} \Sigma_{21}\right)\right)^{2},
$$

where $\lambda_{m}, \ldots, \lambda_{n}$ are the eigenvalues of $\Sigma_{12} \Sigma_{21}$. But

$$
\operatorname{Trace}\left(\Sigma_{12} \Sigma_{21}\right)=\sum_{j=m}^{n} \lambda_{j}=\sum_{i=m}^{n} \sum_{j=n+p+1}^{n+p+k} \sigma_{i j}^{2} \rightarrow 0
$$

as $p \rightarrow \infty$ since $\Sigma \in F_{n}$ for some $n$. Hence, for $p$ large enough, we can write

$$
\left(I_{1}-\Sigma_{12} \Sigma_{21}\right)^{-2}=\sum_{k=0}^{\infty}\left(\begin{array}{c}
-2 \\
k
\end{array}\right)\left(-\Sigma_{12} \Sigma_{21}\right)^{k}=I_{1}+2 \Sigma_{12} \Sigma_{21}+O\left(\left\|\Sigma_{12} \Sigma_{21}\right\|_{F}^{2}\right) .
$$

Then, by (33) and (36),

$$
\operatorname{Trace}\left(\Sigma^{12} \Sigma^{21}\right)=\operatorname{Trace}\left(\Sigma_{12} \Sigma_{21}\right)+2 \operatorname{Trace}\left(\Sigma_{12} \Sigma_{21}\right)^{2}+O\left(\left\|\Sigma_{12} \Sigma_{21}\right\|_{F}^{3}\right) .
$$

In view of (34) and (35), the proof is complete.

\section{Applications to Statistics}

This paper was motivated by the problem of estimating the covariance matrix of $n$ independent identically distributed $p$-vectors, $\mathbf{X}_{1}, \ldots, \mathbf{X}_{N}$ with a common $N\left(\mathbf{0}, \Sigma_{p}\right)$ distribution. In [1,2] Bickel and Levina show that covariance matrices which are approximable by banded matrices could be well estimated, in the operator norm, by banded empirical covariance matrices, and accordingly their inverses could be approximated by the inverses of the estimates above. Conversely, inverse covariance matrices approximable by banded matrices could be well approximated by data dependent banded matrices and now the covariance matrices themselves would be approximated by the inverses of the banded matrices above. The bounds developed above enable us to approximate both a covariance and its inverse by banded matrices simultaneously in a very explicit way.

Specifically consider $\Sigma_{p}$ as the top left $p \times p$ block of a banded matrix $\Sigma: \ell^{2} \rightarrow \ell^{2}$ with a bounded inverse $\Sigma^{-1}$. Let $\|\cdot\|$ be the operator norm and put

$$
\delta_{k}(\Sigma):=\operatorname{dist}\left(\Sigma, \mathrm{BO}_{k}\right)=\min \left\{\|\Sigma-B\|: B \in \mathrm{BO}_{k}\right\}=:\left\|\tilde{B}_{k}(\Sigma)-\Sigma\right\| .
$$

with $\tilde{B}_{k}(\Sigma) \in \mathrm{BO}_{k}$. Theorem 2.3, specialized to A positive definite and self-adjoint, says that

$$
\delta_{n k}\left(\Sigma^{-1}\right) \leq \frac{2 \delta_{k}}{m\left(m-2 \delta_{k}\right)}+\frac{1}{m}\left(\frac{\kappa-1}{\kappa+1}\right)^{n}
$$

where $\delta_{k}=\delta_{k}(\Sigma), m^{-1}=m^{-1}(\Sigma)$ is the norm of $\Sigma^{-1}, M=M(\Sigma)$ is the norm of $\Sigma$ and $\kappa=\kappa(\Sigma)=\frac{M}{m}$ is the condition number of $\Sigma$. Let

$$
\hat{\Sigma}_{p}:=\frac{1}{N} \sum_{i=1}^{N}\left(\mathbf{X}_{i}-\overline{\mathbf{X}}\right)\left(\mathbf{X}_{i}-\overline{\mathbf{X}}\right)^{T}
$$

be the empirical covariance of $\mathbf{X}_{p \times 1}$. The individual elements of $\hat{\Sigma}_{p}, \hat{\sigma}_{i j}$ approach the corresponding $\sigma_{i j}$ as $N \rightarrow \infty$ with high probability but $\hat{\Sigma}_{p}$ fails to have an inverse if $p \geq N$ and its eigenstructure is, in general, diverging from that of $\Sigma$, if $p$ is commensurate or much larger than $N$ i.e. as $p \rightarrow \infty$ as well as 
$N$ with $\frac{p}{N} \rightarrow c, 0<c \leq \infty$, see Johnstone [7]. However, we can, under very mild conditions on $p$ and $N$, find $k_{N} \rightarrow \infty$ such that

$$
\left\|B_{k_{N}}\left(\hat{\Sigma}_{p}\right)-B_{k_{N}}\left(\Sigma_{p}\right)\right\| \rightarrow 0
$$

in probability, where

$$
B_{k}(A):=\left(a_{i j} \chi(|i-j| \leq k)\right)_{i, j=1}^{p}
$$

if $A=\left(a_{i j}\right)_{i, j=1}^{p}$. In particular, for Gaussian $\mathbf{X}$ this is true if $\frac{\log p}{N} \rightarrow 0$. Therefore if $\Sigma \in$ BDO, so that $\delta_{k}(\Sigma) \rightarrow 0$ as $k \rightarrow \infty$, this yields an operator norm consistent estimate of $\Sigma$, that is

$$
\left\|B_{k_{N}}\left(\hat{\Sigma}_{p}\right)-\Sigma_{p}\right\| \leq\left\|B_{k_{N}}\left(\hat{\Sigma}_{p}\right)-B_{k_{N}}\left(\Sigma_{p}\right)\right\|+\left\|B_{k_{N}}\left(\Sigma_{p}\right)-\Sigma_{p}\right\| \stackrel{P}{\longrightarrow} 0 .
$$

We can deduce from (37) that if $\left\|\Sigma^{-1}\right\|<\infty$ then

$$
\left\|B_{n k_{N}}\left(\Sigma_{p}^{-1}\right)-\Sigma_{p}^{-1}\right\| \rightarrow 0
$$

as $n \rightarrow \infty$ slowly with $k_{N}$. It is, of course, clear from (38) and (40) that $\left[B_{k_{N}}\left(\hat{\Sigma}_{p}\right)\right]^{-1}$ will eventually exist, be self-adjoint positive definite and consistently estimate $\Sigma^{-1}$. However, this is rather unsatisfactory in practice as well as theory since $B_{k_{N}}^{-1}\left(\hat{\Sigma}_{p}\right)$ in general does not have a band structure (meaning that it is supported on all its diagonals) in particular if we assume that $\Sigma^{-1} \in \mathrm{BO}$, so that by Theorem 2.3, $\Sigma \in \mathrm{BDO}$, our estimate would not reflect this information. The assumption that $\Sigma_{p}^{-1}$ belongs to $\mathrm{BO}_{k}$ has, in the Gaussian case, a statistical interpretation. It implies that $\mathbf{X}_{i}$ is independent of $\left\{\mathbf{X}_{j}:|i-j|>k\right\}$ given $\left\{\mathbf{X}_{j}:|i-j| \leq k, j \neq i\right\}$. The assumption that $\Sigma_{p} \in \mathrm{BO}_{k}$ has a different interpretation, implying that $\mathbf{X}_{i}$ is independent (unconditionally) of $\left\{\mathbf{X}_{j}:|i-j|>k\right\}$. One of the interesting consequences of Theorem 2.3 is that it tells us that conditional independence (for a band structure) cannot occur unless there is approximate conditional independence and vice versa. That point aside, we are left with a good but not "natural" estimate for $\Sigma_{p}^{-1}$ if we assume $\Sigma^{-1} \in$ BO. However, our approach to Theorem 2.3 tells us precisely what to do.

Suppose $B_{k}\left(\hat{\Sigma}_{p}\right)$ is our estimate of $\Sigma_{p}$. Let $\hat{m}_{k}, \hat{M}_{k}$ be the minimal and maximal absolute eigenvalues of $B_{k}\left(\hat{\Sigma}_{p}\right)$ and

$$
\hat{\gamma}:=2\left(\hat{M}_{k}+\hat{m}_{k}\right)^{-1}
$$

Then an $n k$ banded estimate of $\Sigma_{p}^{-1}$ is just

$$
\hat{\gamma} \sum_{j=0}^{n}\left(I-\hat{\gamma} B_{k}\left(\hat{\Sigma}_{p}\right)\right)^{j} .
$$

We are left with the problem of how to choose $k$ and $n$. In theory, if we have some notion or make assumptions about the magnitude of the "bias" $\delta_{k}(\Sigma)$ and calculate stochastic bounds on the "variance" $\left\|B_{k}\left(\hat{\Sigma}_{p}\right)-B_{k}\left(\Sigma_{p}\right)\right\|$ and make assumptions about how many zeros $\Sigma^{-1}$ has, we can use (37) to estimate the optimal choices of $k$ and $n$. In practice, it is better to use some data determined choice, e.g. by crossvalidation, see Bickel, Levina [1,2]. However, we believe that (37) and (39) can be used to compute minmax bounds and oracle inequalities on the performance of estimates of $\Sigma_{p}^{-1}$, from the ones obtained for estimates of $\Sigma_{p}$, see Cai, Zhang, Zhou [3]. It should be clear that whatever we have said of banding applies to generalized banding up to a permutation also.

The application of extended generalized banding is satisfactory if we think of the coordinates of $\mathbf{X}$ as corresponding to labelled points on a manifold, as is reasonable in (say) geophysical applications, where $\mathbf{X}$ is the state of some variable such as pressure, at a grid of points on the globe at some time. But it is not relevant if the labels are meaningless as in microarrays genomics, where the coordinates simply label genes. However, it should now be clear that our second generalization to generalized banding up to some unknown permutation $\pi$ deals with such situations. It enables us to define classes of matrices $\Sigma$ in terms of their dependency graph defined in terms of $\Sigma^{-1}$ by having vertices correspond to coordinates of $\mathbf{X}$ with an edge between $i$ and $j$ if the $(i, j)$ th entry of $\Sigma^{-1}$ is different from 0 . However, although the determination of $\varrho$ is dictated by the situation, estimation of $\pi$ is nontrivial and will be pursued elsewhere. 
Acknowledgements. This research was partially supported by NSF grants DMS-0605236 and DMS0906808 to Bickel and by Marie Curie Grants MEIF-CT-2005-009758 and PERG02-GA-2007-224761 to Lindner. Both authors have been supported by the Statistics and Applied Mathematics Institute (NSF) and the American Institute of Mathematics (AIM).

\section{References}

[1] P. Bickel and E. Levina: Regularized Estimation of Large Covariance Matrices, Annals of Statistics 36 (2008), 199-227.

[2] P. Bickel and E. Levina: Covariance Regularization by Thresholding, Annals of Statistics, 36 (2008), 2577-2604.

[3] T. Cai, C.H. Zhang and H. Zhou: Optimal Rates of Convergence for Covariance Matrix Esti-

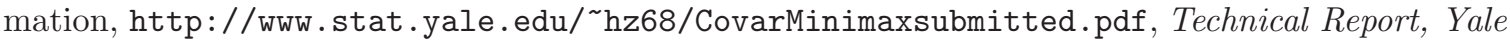
University, 2008.

[4] J. Dixmier: $C^{*}$-Algebras, North Holland Publ. Comp., Amsterdam, New York, Oxford 1982.

[5] I. A. Ibragimov and Yu. A. Rozanov: Gaussian Random Processes, Springer-Verlag, New York, 1978.

[6] I. A. Ibragimov and V. N. Solev: A condition for regularity of a Gaussian stationary process, Soviet Math. Doklady 10 (1969), 371-375.

[7] I.M. Johnstone: High dimensional statistical inference and random matrices. International Congress of Mathematicians Vol.I, 307-333, Eur. Math. Soc., Zurich, 2007.

[8] A.N. Kolmogorov and Y.A. Rozanov: On the strong mixing conditions of a stationary Gaussian process. Th. Prob. Applications 5, 1960, 204-208.

[9] V. G. Kurbatov: The algebra of difference operators, Manuscript No. 1017-82, deposited at VINITI, 1982 (Russian), R. Zh. Mat. 1982, $7 \mathrm{~b} 767$.

[10] V. G. Kurbatov: On the invertibility of almost periodic operators, Math USSR Sbornik, 67 (1990), $367-377$.

[11] V. G. Kurbatov: Functional Differential Operators and Equations, Kluwer Academic Publishers, Dordrecht, Boston, London 1999.

[12] B. V. LANGE and V. S. Rabinovich: The algebra of generalized discrete convolution operators with oscillating coefficients, Manuscript No. 3661-82, deposited at VINITI, 1982. (Russian) R. Zh. Mat. 1982, $12 \mathrm{~b} 972$.

[13] S. Z. LeVendorski and V. S. Rabinovich: Exponential decrease at infinity of solutions of multidimensional convolution equations in cones, Izv. Vyssh. Uchebn. Zaved. Mat. 202 (1979), no. 3, 38-44, Engl. transl. in Soviet Math. (Iz. VUZ) 23 (1979).

[14] M. LindneR: Infinite Matrices and their Finite Sections: An Introduction to the Limit Operator Method, Frontiers in Mathematics, Birkhäuser 2006.

[15] M. LindneR: Independence of Fredholmness and index on the underlying space for operators in the Wiener algebra, Journal of Operators and Matrices 2 (2008), 297-306.

[16] Ya. A. Lutski and V. S. Rabinovich: Pseudodifferential operators in spaces of generalized functions with exponential behaviour at infinity, Funktsional. Anal. i Prilozhen. 12 (1978), no. 1, 79; Engl. transl. in Funct. Anal. Appl. 12 (1978). 
[17] V. S. Rabinovich, S. Roch and B. Silbermann: Limit Operators and Their Applications in Operator Theory, Birkhäuser 2004.

[18] T. Runst and W. Sickel: Sobolev Spaces of Fractional Order, Nemytskij Operators, and Nonlinear Partial Differential Equations, de Gruyter 1996.

[19] M. A. Shubin: Almost periodic functions and partial differential operators, Russian Math. Surv. 33 (1978), 1-52.

[20] M. A. Shubin: Pseudodifferential operators and their Green's functions, Izv. Akad. Nauk SSSR Ser. Mat. 49 (1985), 652-671; Engl. transl. in Math. USSR Izv. 26 (1986).

\section{Author's addresses:}

Peter J. Bickel

bickel@stat.berkeley.edu

Department of Statistics

367 Evans Hall

Berkeley, CA, 94710-3860

USA

Marko Lindner

Fakultät Mathematik

TU Chemnitz

D-09107 Chemnitz

GERMANY marko.lindner@mathematik.tu-chemnitz.de (corresponding author) 